\section{FRI0524 HUMAN MONOCLONAL ACPAS INDUCE MOBILITY OF PRIMED SYNOVIAL FIBROBLAST IN A PAD- DEPENDENT PATHWAY}

Meng Sun, Akilan Krishnamurthy, Alexandra Circiumaru, Marianne Engström, Johanna Steen, Philip Titcombe, Vivianne Malmström, Heidi Wähämaa, Lars Klareskog, Bence Réthi, Anca Catrina. Karolinska Institutet and Univerisity Hospital, Rheumatology Unit, Department of Medicine, Stockholm, Sweden

Background: Anti-citrullinated protein antibodies (ACPA) play an important role in rheumatoid arthritis (RA) pathogenesis. They recognize a wide number of citrullinated targets and show limited cross-reactivity to acetylated ones(1).

Objectives: We aimed to investigate the effect of monoclonal ACPAs with different binding patterns on RA synovial tissue derived fibroblasts (SF). Methods: Synovial fibroblasts were isolated from synovial tissue of RA patients by enzymatic digestion. ACPA and control monoclonal antibodies (mAbs) were derived from single $B$ cells isolated from RA patients. Two monoclonal ACPA (1325:01B09 and 1325:05C06) showed cross-reactivity to acetylated-targets while four of them (1325:04C03, 1325:07E07, 14CFCT2D09, 14CFCT2H12) did not(1). mAbs were tested in synovial fibroblast migration (Incucyte live time image analysis) and osteoclast formation (TRAP) assays. The role of mAbs cross-reactivity was tested in fibroblast migration assays using inhibitors of peptidylarginine deiminases (Cl-amidine), histone acetyltransferase (anacardic acid) and histone deacetylase (trichostatin A). Binding patterns of monoclonal ACPAs were tested in synovial biopsies obtained from both healthy donors and RA patients. Results: One acetylation cross-reactive ACPA (1325:01B09) and two with no cross reactivity (14CFCT2D09 14CFCT2H12) significantly enhanced fibroblast migration (mean $\pm \mathrm{SD}$ fold increase of $1.9 \pm 0.5,1.7 \pm 0.4$ and $1.8 \pm 0.6$, respectively) compared to control mAb 1362:01E02 ( $p<0.05)$, while having no effect on osteoclast formation. In contrast, one acetylation non-cross-reactive ACPA (1325:04C03) promoted osteoclastogenesis (mean \pm SD fold increase of 1.6 $\pm 0.05)$ compared to control mAb 1362:01E02 $(p<0.05)$, while having no effect on fibroblast migration. Cl-amidine completely abolished the effect of 1325:01B09, while neither anacardic acid nor trichostatin A had any effect. Moreover, the fibroblast-promoting ACPA (1325:01B09) but not the osteoclastogenic ACPA1325:04C03 co-localize with CD55-positive SF in the inflamed rheumatoid synovium. No detectable signals were found in healthy synovium. Conclusion: Monoclonal ACPAs have distinct cellular effects on synovial fibroblasts and osteoclasts that are not related to cross reactivity towards acetylated targets

\section{REFERENCES:}

[1] K. A. Lloyd, G. Wigerblad, P. Sahlstrom, M. G. Garimella, K. Chemin, J. Steen, P. J. Titcombe, B. Marklein, D. Zhou, R. Stalesen, E. Ossipova, C. Lundqvist, O. Ekwall, J. Ronnelid, D. L. Mueller, M. C. I. Karlsson, M. J. Kaplan, K. Skriner, L. Klareskog, F. Wermeling, V. Malmstrom, C. Gronwall, Differential ACPA Binding to Nuclear Antigens Reveals a PAD-Independent Pathway and a Distinct Subset of Acetylation Cross-Reactive Autoantibodies in Rheumatoid Arthritis. Front Immunol 9, 3033 (2018).

Disclosure of Interests: Meng Sun Grant/research support from: Yes, but not for presented project., Akilan Krishnamurthy: None declared, Alexandra Circiumaru: None declared, Marianne Engström: None declared, Johanna Steen: None declared, Philip Titcombe: None declared, Vivianne Malmström: None declared, Heidi Wähämaa: None declared, Lars Klareskog Grant/research support from: Yes, but not for the presented study., Bence Réthi: None declared, Anca Catrina Grant/research support from: Yes, but not for the presented study.

DOI: 10.1136/annrheumdis-2019-eular.4821

\section{VISAFTIN-MEDIATED OSTEODESTRUCTIVE EFFECTS ARE REDUCED}

\section{FRI0525 DURING ADIPOGENIC DIFFERENTIATION OF MSC ON MINERALIZED BONE FRAGMENTS}

Lali Tsiklauri ${ }^{1}$, Janina Werner ${ }^{2}$, Klaus Frommer ${ }^{1}$, Stefan Rehart ${ }^{3}$, Sabine Wenisch ${ }^{2}$, Ulf Müller-Ladner ${ }^{1}$, Elena Neumann ${ }^{1} .{ }^{1}$ Justus-Liebig-University Giessen, Department of Internal Medicine and Rheumatology, Kerckhoff-Klinik, Bad Nauheim, Germany, Bad Nauheim, Germany; ${ }^{2}$ Institute of VeterinaryAnatomy, -Histology and -Embryology, Clinic of Small Animals, JLU Giessen, Germany, Giessen, Germany; ${ }^{3}$ Markus Hospital, Frankfurt, Germany, Department of Orthopedics and Trauma Surgery, Frankfurt, Germany

Background: Osteoporosis (OP), as an age-related disease, is characterized by bone loss, increased fracture risk and poor regeneration. During aging and OP bone marrow adiposity is increased due to a shift of osteogenic towards adipogenic differentiation of bone marrow mesenchymal stem cells (MSC). The differentiation of MSC into adipocytes or osteoblasts is an important determinant of bone structural integrity. It is known, that fatty tissue not simply functions as energy storage but is metabolically highly active. Therefore, adipocyte-derived factors such as adipokines might influence MSC differentiation.

Objectives: The role of interactions between adipocyte-derived factors and MSC in the pathogenesis of OP is not fully elucidated. Thus, we analyzed the presence of the adipokine visfatin in bone tissue and its effects on MSC differentiation in standard culture vs. spongiosa.

Methods: The spongiosa of femoral heads of patients with osteoarthritis (OA) after hip replacement surgery, or after osteoporotic femoral neck fracture were used for RNA- and MSC-isolation. Adipogenic MSC differentiation was performed with/without visfatin and its inhibitor Apo866 as well as SB203580 p38-MAPK inhibitor. For the transfer and differentiation of MSC on cancellous bone, bone fragments were purified and sterilized. Gene expression was measured by Realtime PCR. Protein production was evaluated by ELISA.

Results: Elevated visfatin-level were observed in OP compared to nonosteoporotic OA bone. Visfatin-induced secretion of proinflammatory factors was lower during adipogenesis on cancellous bone then in standard cell culture (e.g. 14d IL6, x-fold: standard culture 151 \pm 110 , spong. 40 \pm 30 , $\mathrm{n}=7$ ). Significantly elevated MMP13 mRNA as well as protein expression induced by visfatin could be detected during adipogenesis on spongiosa as well as in standard cell culture. However visfatin-mediated MMP13 expression was markedly reduced in the presence of cancellous bone (e. g. 21 d, $x$-fold: standard culture $81 \pm 89$, spong. $13 \pm 21, n=7$ ). Inhibition of visfatin by Apo866 decreased the visfatin-induced cytokine release but not the MMP13 expression during adipogenesis in culture $(n=4)$. In contrast to Apo866, the p38-MAPK inhibitor did not influence cytokine release but reduced MMP13 expression in a time dependent manner. Conclusion: Visfatin level was elevated in osteoporotic vs OA bone. Therefore, visfatin-mediated increase of MMPs and proinflammatory cytokines during adipogenic differentiation might influence bone turnover at the adipose tissue/bone interface. Our results support the idea that the extracellular matrix attenuates visfatin-mediated detrimental effects during adipogenesis. The observed visfatin-mediated effects most likely depend on different signaling pathways.

Disclosure of Interests: Lali Tsiklauri: None declared, Janina Werner: None declared, Klaus Frommer: None declared, Stefan Rehart: None declared, Sabine Wenisch: None declared, Ulf Müller-Ladner Grant/ research support from: supported by an unrestricted educational grant from Celgene $\mathrm{GmbH}$., Elena Neumann: None declared DOI: 10.1136/annrheumdis-2019-eular.4328

\section{FRI0526 IL37 AMELIORATES EXPERIMENTAL MURINE OSTEOARTHRITIS}

Arjan van Caam, Ellen van Geffen, Joyce Aarts, Elly Vitters, Esmeralda Blaney Davidson, Peter van der Kraan. Radboud university medical center, Experimental Rheumatology, Nijmegen, Netherlands

Background: Osteoarthritis $(\mathrm{OA})$ is the world's most common degenerative joint disorder and leads to pain and disability. Currently, no disease-modifying drugs are available, resulting in a large unmet clinical need Recently, we have shown that the anti-inflammatory cytokine IL37 lowers pro-inflammatory cytokine production, MMP3 activity and sulfated glycosaminoglycan loss from ex vivo cultured chondrocytes and human cartilage explants. In this study, we further explored the ability of IL37 to ameliorate OA pathology by studying its effects both in vitro on synovial fibroblasts and in vivo in collagenase-induced $\mathrm{OA}$

Objectives: To establish if IL37 can ameliorate experimental OA pathology

Methods: Primary synovial fibroblasts were obtained from synovial biopsies of 10 patients undergoing total joint replacement surgery. IL37 was overexpressed in these cells using an adenovirus and subsequently cells were challenged with IL1 $\beta$ or OA-synovium conditioned medium. Collagenase-induced $\mathrm{OA}$ (CiOA) was induced by injecting 10 weeks old female C57BL/6J with a single intra-articular injection of 3 units of collagenase type VII. Four days after collagenase injection, Ad-IL37 or Ad-Luc (= control) was intra-articular injected in the right knee joint of mice. Two weeks later this injection was repeated. Mice were sacrificed on day 7 , 28 and 42 after collagenase-injection. Synovial thickening, cartilage damage and osteophyte formation were measured histologically. IL37, MMP3 and VDIPEN expression were investigated by immunohistochemistry. 